\title{
Advection Dispersion Equation and BMO Space
}

\author{
Kan Zhang ${ }^{1,2 *}$, Tieliang Wang ${ }^{1 \#}$, Xue Feng ${ }^{2}$ \\ ${ }^{1}$ Postdoctoral of Agricultural Engineering, Shenyang Agricultural University, Shenyang, China \\ ${ }^{2}$ College of Sciences, Shenyang Agricultural University, Shenyang, China \\ Email: kanzhang2004@163.com, ”wzhangpaper@163.com, xfeng2000@163.com
}

Received October 15, 2013; revised November 10, 2013; accepted November 14, 2013

Copyright (C) 2013 Kan Zhang et al. This is an open access article distributed under the Creative Commons Attribution License, which permits unrestricted use, distribution, and reproduction in any medium, provided the original work is properly cited.

\section{ABSTRACT}

In this paper, we provide a new way of characterizing the upper and lower bound for the concentration and the gradient of concentration in advection dispersion equation under the condition that source term, concentration and stirring term belong to BMO space.

Keywords: Advection Dispersion Equation; BMO; Concentration

\section{Introduction}

Throughout the paper we fix a positive integer $n$ and let

$$
C^{n}=C \times \cdots \times C
$$

denote the $n$-dimensional complex Euclidean space. For $z=\left(z_{1}, z_{2}, \cdots, z_{n}\right)$ and $\omega=\left(\omega_{1}, \omega_{2}, \cdots, \omega_{n}\right)$ in $C^{n}$ we write

$$
|z|^{2}=\sum_{i=1}^{n}\left|z_{i}\right|^{2}
$$

and

$$
\langle z, \omega\rangle=z_{1} \overline{\omega_{1}}+\cdots+z_{n} \overline{\omega_{n}},
$$

where $\overline{\omega_{i}}$ is the complex conjugate of $\omega_{i}$.

Let $B_{n}$ denote the unit ball in $C^{n}$ and let $v$ be the Lebesgue volume measure on $B_{n}$. For $-1<\alpha<\infty$, we denote by $v_{\alpha}$ the measure on $B_{n}$ defined by

$$
\mathrm{d} v_{\alpha}(z)=c_{\alpha}\left(1-|z|^{2}\right)^{\alpha} \mathrm{d} v(z) \text {, where } c_{\alpha}=\frac{\Gamma(n+\alpha+1)}{n ! \Gamma(\alpha+1)}
$$

is a normalizing constant such that $v_{\alpha}\left(B_{n}\right)=1$. For $1 \leq p<\infty$, we write \|\|$_{\alpha, p}$ for the norm on $L^{p}\left(B_{n}, \mathrm{~d} v_{\alpha}\right)$ and $\langle\cdot \cdot\rangle_{\alpha}$ for the inner product on $L^{2}\left(B_{n}, \mathrm{~d} v_{\alpha}\right)$. The weighted Bergman space $A_{\alpha}^{p}\left(B_{n}\right)$ consists of holomorphic functions $f$ in $L^{p}\left(B_{n}, \mathrm{~d} v_{\alpha}\right)$, that is,

\footnotetext{
*The first author is partly supported by NSFC, Item Number: 11371182.

"Corresponding author.
}

$$
A_{\alpha}^{p}\left(B_{n}\right)=L^{p}\left(B_{n}, \mathrm{~d} v_{\alpha}\right) \cap H\left(B_{n}\right),
$$

where $H\left(B_{n}\right)$ denote the space of all holomorphic functions in $B_{n}$. Reproducing kernels $K_{w}^{\alpha}$ and normalized reproducing kernels $k_{w}^{\alpha}$ in $A_{\alpha}^{2}\left(B_{n}\right)$ are given by, respectively,

$$
K_{w}^{\alpha}(z)=\frac{1}{(1-\langle z, w\rangle)^{n+\alpha+1}}
$$

and

$$
k_{w}^{\alpha}(z)=\frac{\left(1-|w|^{2}\right)^{(n+\alpha+1) / 2}}{(1-\langle z, w\rangle)^{n+\alpha+1}}
$$

for $z, w \in B_{n}$. For every $h \in A_{\alpha}^{2}\left(B_{n}\right)$ we have $\left\langle h, K_{w}^{\alpha}\right\rangle_{\alpha}=h(w)$ for all $w \in B_{n}$. The orthogonal projection $P_{\alpha}$ of $L^{2}\left(B_{n}, \mathrm{~d} v_{\alpha}\right)$ onto $A_{\alpha}^{2}\left(B_{n}\right)$ is given by

$$
\left(P_{\alpha} g\right)(w)=\left\langle g, K_{w}^{\alpha}\right\rangle_{\alpha}=\int_{B_{n}} g(z) \frac{1}{(1-\langle w, z\rangle)^{n+\alpha+1}} \mathrm{~d} v_{\alpha}(z)
$$

for $g \in L^{2}\left(B_{n}, \mathrm{~d} v_{\alpha}\right)$ and $w \in B_{n}$. When $n=1$, we write $D$ for $B_{1}$.

For $f \in L^{1}\left(B_{n}, \mathrm{~d} v_{\alpha}\right)$, we define the Berezin transform of $f$ to be the function $\tilde{f}$, that is

$$
\tilde{f}(z)=\int_{B_{n}} f(w)\left|k_{z}^{\alpha}(w)\right|^{2} \mathrm{~d} v_{\alpha}(w) .
$$

If $f$ is bounded, then $\tilde{f}$ is a bounded function on $B_{n}$. Since the normalized reproducing kernels $k_{z}^{\alpha}$ converge weakly to zero as $z$ tends $\partial B_{n}$, we have that if 
$f$ is compact, then $\tilde{f}(z) \rightarrow 0$ as $z \rightarrow \partial B_{n}$. The converse (in both case) is not necessarily true.

The traditional advection dispersion equation is a standard model for contaminant transport. The advection dispersion equation is the basis of many physical and chemical phenomena, and its use has also spread into economics, financial forecasting and other fields. In general, the numerical solution of advection dispersion equations has been dominated by either finite difference, finite element or boundary element methods. These methods are derived from local interpolation schemes and require a mesh to support the application. It is well known that the numerical solution of advection dispersion equation is a difficult task. Scholars try to find the new way to obtain the solution of advection dispersion equation. For the passive scalar, complicated behavior is often observed even for laminar velocity fields. This is the well-known effect of chaotic advection in $[1,2]$. Thus we can choose the source and stirring term of the advection dispersion equation to be any divergence-free, possibly time-dependent flow field. The mixing efficiency depends on specific properties of the stirring and source term. Schumacher, Sreenivasan and Yeung have obtained bounds on high-order derivative moments of a passive scalar for large values of the Schmidt number in [3]. Thiffeault, Doering and Gibbon have obtained bounds on mixing efficiency for the passive scalar under the influence of advection and diffusion with a body source in [4].

The advection dispersion equation for the concentration $\theta(x, t)$ of a passive scalar is

$$
\frac{\partial \theta}{\partial t}+u \cdot \nabla \theta=k \Delta \theta+s
$$

where $k$ is the dispersion coefficient, $s(x, t)$ is a source term, and $u(x, t)$ is the stirring term. It is clear that an exciting mixing configuration would have small concentration for a given source term and stirring term, indicating a steady state with low density of concentration. We use the fluctuations in the concentration as a useful measure of the degree of well-mixedness, as has long been the practice in $[5,6]$.

In this paper we apply some recent developments in the analysis of the BMO space to the advection dispersion equation. We further provide a new way of giving the upper and lower bound for the concentration and the gradient of concentration in the advection dispersion equation by using BMO theory. The bounds on mixing efficiency in this paper mainly depend on the stirring field and the source distribution, which is very important for allowing comparison of the relative effectiveness of various source term for specified stirring scenarios.

Throughout the paper, we will use the letter $c$ to de- note a generic positive constant that can change its value at each occurrence.

\section{Some Lemmas and Basic Definitions}

For $z \in B_{n}$, let $\psi_{z}$ be the automorphism of $B_{n}$ such that $\psi_{z}(0)=z$ and $\psi_{z}=\psi_{z}^{-1}$, which is described in [7]. It has the real Jacobian equal to

$$
\left|\psi_{z}^{\prime}(w)\right|^{2}=\frac{\left(1-|z|^{2}\right)^{n+1}}{|1-\langle z, w\rangle|^{2 n+2}},
$$

for $z, w \in B_{n}$.

Thus we have the change-of-variable formula

$$
\int_{B_{n}} h\left(\psi_{z}(w)\right)\left|k_{z}^{\alpha}(w)\right|^{2} \mathrm{~d} v_{\alpha}(w)=\int_{B_{n}} h(w) \mathrm{d} v_{\alpha}(w),
$$

for every $h \in L^{1}\left(B_{n}, \mathrm{~d} v_{\alpha}\right)$.

By a well-known theorem of John-Nirenberg in $[8,9]$, the classical BMO of the unit circle is independent of the $L^{p}$ norm used to define it. It is also well known that a function $f$ on the circle is in BMO if and only if the Hankel operators with symbol $f$ and $\bar{f}$ are both bounded on the Hardy space of the circle. A new type of BMO, Denoted $B M O_{\partial}(\Omega)$, is introduced in $[10,11]$ for any bounded domain $\Omega$ in the complex space $C^{n}$. In this paper we define the BMO space in the Bergman metric by the $L^{p}$ norm.

Let $f \in L^{1}\left(B_{n}, \mathrm{~d} v_{\alpha}\right)$ and $p \geq 1$, we say that $f \in B M O_{\alpha}^{p}\left(B_{n}\right)$ whenever

$$
\|f\|_{B M O_{\alpha}^{p}}=\sup _{z \in B_{n}}\left\|f \circ \psi_{z}-\tilde{f}(z)\right\|_{\alpha, p}<\infty .
$$

Note that \|\|$_{B M O_{\alpha}^{p}}$ does not distinguish constants, while $\|f\|_{\alpha, p}=\|f\|_{B м O_{\alpha}^{p}}+|\tilde{f}(0)|$ is a norm in $B M O_{\alpha}^{p}\left(B_{n}\right)$. By the Theorem 5 in [12], we know the fact that $B M O_{\alpha}^{p}\left(B_{n}\right)$ is equivalent to $B M O_{r}^{p}$ in [12]. For $z, w \in B_{n}$, let $\beta(z, w)=\frac{1}{2} \log \frac{1+\left|\psi_{z}(w)\right|}{1-\left|\psi_{z}(w)\right|}$ denote the Bergman metric on $B_{n}$. For any $z \in B_{n}$ and $r>0$, let

$$
D(z)=\left\{w \in B_{n} \mid \beta(z, w)<r\right\}
$$

be the Bergman metric ball with center $z$ and radius $r$. Let $|D(z)|=v_{\alpha}(D(z))$, which is equivalent to $\left(1-|z|^{2}\right)^{n+\alpha+1}$ (see Lemma 1.24 of [13]).

For $f \in L^{1}\left(B_{n}, \mathrm{~d} v_{\alpha}\right)$, the average of $f$ over $D(z)$ is defined by 


$$
\hat{f}(z)=\frac{1}{|D(z)|} \int_{D(z)} f(w) \mathrm{d} v_{\alpha}(w) .
$$

Using the properties of Bergman metric, it follows that

$$
\sup _{z \in B_{n}} \frac{1}{|D(z)|} \int_{D(z)}|f(w)-\hat{f}(z)|^{p} \mathrm{~d} v_{\alpha}(w)<\infty
$$

if and only if $\|f\|_{\text {Вмо }}<\infty$.

Thus, functions in $B M O_{\alpha}^{p}\left(B_{n}\right)$ have bounded mean oscillation in the Bergman metric. Since $B M O_{\alpha}^{p}\left(B_{n}\right)$ functions are locally in $L^{p}\left(B_{n}, \mathrm{~d} v_{\alpha}\right)$, it is not hard to see that

$$
\begin{array}{cl}
L^{\infty}\left(B_{n}\right) \subset B M O_{\alpha}^{p}\left(B_{n}\right) \subset L^{p}\left(B_{n}, \mathrm{~d} v_{\alpha}\right), & p \geq 1, \\
B M O_{\alpha}^{q}\left(B_{n}\right) \subset B M O_{\alpha}^{p}\left(B_{n}\right) \subset B M O_{\alpha}^{1}\left(B_{n}\right), & 1 \leq p \leq q .
\end{array}
$$

Since the space $B M O_{\alpha}^{1}\left(B_{n}\right)$ is the largest among the $B M O_{\alpha}^{p}\left(B_{n}\right)$ for $p \geq 1$, from now on we will be mainly interested in functions belonging to this class. The study of BMO spaces plays an important role on modern analysis and applied science in [14,15]. For simplicity we will write $B M O_{\alpha}^{p}$ instead of $B M O_{\alpha}^{p}\left(B_{n}\right) . V M O_{\alpha}^{p}$ consists of functions $f$ in $B M O_{\alpha}^{p}$ such that

$\left\|f \circ \psi_{z}-\tilde{f}(z)\right\|_{\alpha, p} \rightarrow 0$ as $|z| \rightarrow 1^{-}$.

Lemma 2.1 Suppose $f \in B M O_{\alpha}^{1}$, then the following quantities are equivalent:

(1) $\hat{f}$ is bounded on $B_{n}$;

(2) $\tilde{f}$ is bounded on $B_{n}$;

(3) $\widetilde{|f|}$ is bounded on $B_{n}$;

(4) $|\widehat{f}|$ is bounded on $B_{n}$.

Proof

$$
\begin{aligned}
(1) \Leftrightarrow & (2) \text { : Since } \\
& |\tilde{f}(z)-\hat{f}(z)| \\
& =\left|\frac{1}{\mid D(z)} \int_{D(z)}(\tilde{f}(z)-f(\omega)) \mathrm{d} v_{\alpha}(\omega)\right| \\
& \leq \frac{1}{|D(z)|} \int_{D(z)}|\tilde{f}(z)-f(\omega)| \mathrm{d} v_{\alpha}(\omega) \\
& \leq c \int_{B_{n}}|\tilde{f}(z)-f(\omega)|\left|k_{z}^{\alpha}(\omega)\right|^{2} \mathrm{~d} v_{\alpha}(\omega) \\
& =c \int_{B_{n}}\left(\left|\tilde{f}(z)-f \circ \psi_{z}(\omega)\right|\right) \mathrm{d} v_{\alpha}(\omega) \\
& \leq c\|f\|_{B M O_{\alpha}^{1}},
\end{aligned}
$$

then $\hat{f}$ is bounded on $B_{n}$ if and only if $\tilde{f}$ is bounded on $B_{n}$.

$$
(2) \Leftrightarrow(3) \text { : Since }
$$

$$
\begin{aligned}
& \widetilde{\mid f}((z)-|\tilde{f}(z)| \\
& =\int_{B_{n}}(|f(\omega)|-|\tilde{f}(z)| \mid)\left|k_{z}^{\alpha}(\omega)\right|^{2} \mathrm{~d} v_{\alpha}(\omega) \\
& \leq \int_{B_{n}}|\tilde{f}(z)-f(\omega)|\left|k_{z}^{\alpha}(\omega)\right|^{2} \mathrm{~d} v_{\alpha}(\omega) \\
& =\int_{B_{n}}\left|\tilde{f}(z)-f \circ \psi_{z}(\omega)\right| \mathrm{d} v_{\alpha}(\omega) \\
& =\|f\|_{B M O_{\alpha}^{1}},
\end{aligned}
$$

then $\tilde{f}$ is bounded on $B_{n}$ if and only if $|\widetilde{f}|$ is bounded on $B_{n}$.

$(3) \Leftrightarrow(4)$ : The proof is trivial.

Lemma 2.1 implies the fact that we may regard the average function and Berezin transform as positive function, which is very important for us to research the parameters in advection dispersion equation by using BMO theory.

\section{Main Results}

In this section, we further obtain the upper and lower bound for the concentration and the gradient of concentration in the advection dispersion equation. We give the reasons why the source term, stirring term and concentration in the advection dispersion equation belong to BMO space. In fact, BMO space extends the meanvariance theory. According to the definition of average function and norm of BMO space, it is clear that the average function extends the mean theory and the norm of BMO space extends the variance theory. The parameters in advection dispersion equation are uniformly bounded in time, which is true under the physical assumption that $\|s\|_{\alpha, 1}$ is uniformly bounded in time. In addition, Lemma 2.1 provides the reasons for regarding the concentration, source term and stirring term as BMO function. The formula

$$
L^{\infty}\left(B_{n}\right) \subset B M O_{\alpha}^{p}\left(B_{n}\right) \subset L^{p}\left(B_{n}, \mathrm{~d} v_{\alpha}\right), \quad p \geq 1,
$$

also provides the reason for $\theta, u, s \in B M O_{\alpha}^{1}$.

In [4], the advection dispersion operator is defined by

$$
L=\frac{\partial}{\partial t}+u \cdot \nabla-k \Delta .
$$

It is well known that the space $L^{1}\left(B_{n}, \mathrm{~d} v_{\alpha}\right)$ is Banach space instead of Hilbert space. So it is difficult to obtain the adjoint of the advection dispersion operator. Then we have to find new way of characterizing the upper and lower bound for the concentration and the gradient of concentration in the advection dispersion equation by using BMO theory.

Next, we will obtain the lower bound for $\|\theta\|_{\alpha, 1}$ and $\|\nabla \theta\|_{\alpha, 1}$ in the advection dispersion equation. 
For an arbitrary smooth noralized function $\phi$,

$$
\begin{aligned}
\|s\|_{\alpha, 1}= & \left\|\frac{\partial \theta}{\partial t}+u \cdot \nabla \theta-k \Delta \theta\right\|_{\alpha, 1}=\|L(\theta)\|_{\alpha, 1} \\
\leq & \|L\|_{\alpha, 1}\|\theta\|_{\alpha, 1}=\sup _{\|\phi\|_{\alpha, 1}=1}\left\|\frac{\partial \phi}{\partial t}+u \cdot \nabla \phi-k \Delta \phi\right\|_{\alpha, 1}\|\theta\|_{\alpha, 1} \\
\leq & {\left[\sup _{\|\phi\|_{\alpha, 1}=1}\left\|\frac{\partial \phi}{\partial t}\right\|_{\alpha, 1}+\|u\|_{\alpha, 1} \sup _{\|\phi\|_{\alpha, 1}=1}\|\nabla \phi\|_{\alpha, 1}\right.} \\
& \left.+|k|_{\|\phi\|_{\alpha, 1}=1}\|\Delta \phi\|_{\alpha, 1}\right]\|\theta\|_{\alpha, 1} .
\end{aligned}
$$

So

$$
\geq \frac{\|\theta\|_{\alpha, 1}}{\sup _{\|\left.\phi\right|_{\alpha, 1}=1}\left\|\frac{\partial \phi}{\partial t}\right\|_{\alpha, 1}+\|u\|_{\alpha, 1} \sup _{\|\left.\phi\right|_{\alpha, 1}=1}\|\nabla \phi\|_{\alpha, 1}+|k|_{\|\phi\|_{\alpha, 1}=1}\|\Delta \phi\|_{\alpha, 1}} .
$$

By the formula (3), the lower bound of concentration is proportional to the source term and is inversely proportional to the stirring term and dispersion coefficient, holding the other parameters constant. We still have the freedom to choose $\phi$ to optimize the lower bound of the concentration for a particular problem, that is, for given source term, dispersion coefficient and stirring term.

By the poincare's inequality and the fact

$$
\|s\|_{\alpha, 1}=\left\|\frac{\partial \theta}{\partial t}+u \cdot \nabla \theta-k \Delta \theta\right\|_{\alpha, 1}=\|L(\theta)\|_{\alpha, 1},
$$

we have

$$
\begin{aligned}
\|L\|_{\alpha, 1} & =\sup _{\left\|\theta-\theta_{0}\right\|_{\alpha, 1} \neq 0} \frac{\left\|L\left(\theta-\theta_{0}\right)\right\|_{\alpha, 1}}{\left\|\theta-\theta_{0}\right\|_{\alpha, 1}} \geq c \sup _{\left\|\theta-\theta_{0}\right\|_{\alpha, 1} \neq 0} \frac{\|L(\theta)\|_{\alpha, 1}}{\|\nabla \theta\|_{\alpha, 1}} \\
& \geq c \frac{\|L(\theta)\|_{\alpha, 1}}{\|\nabla \theta\|_{\alpha, 1}}=c \frac{\|s\|_{\alpha, 1}}{\|\nabla \theta\|_{\alpha, 1}} .
\end{aligned}
$$

where $\theta_{0}$, denoted the average concentration of the research domain, is positive constant.

By the formula (4), we obtain

$$
\begin{aligned}
& \|\nabla \theta\|_{\alpha, 1} \geq c \frac{\|s\|_{\alpha, 1}}{\|L\|_{\alpha, 1}} \\
& \geq \frac{c\|s\|_{\alpha, 1}}{\sup _{\|\phi\|_{\alpha, 1}=1}\left\|\frac{\partial \phi}{\partial t}\right\|_{\alpha, 1}+\|u\|_{\alpha, 1} \sup _{\|\phi\|_{\alpha, 1}=1}\|\nabla \phi\|_{\alpha, 1}+|k|_{\|\phi\|_{\alpha, 1}=1}\|\Delta \phi\|_{\alpha, 1}} .
\end{aligned}
$$

concentration is proportional to the source term and is inversely proportional to the stirring term and dispersion coefficient, holding the other parameters constant. We still have the freedom to choose $\phi$ to optimize the lower bound for the gradient of concentration for given source term, dispersion coefficient and stirring term. The formula (5) is true under the condition that the average source term $\hat{s}$ is bounded on $B_{n}$. In other words, the formula (5) does not necessarily hold for emergencies.

Next, we will obtain the upper bound for $\|\theta\|_{\alpha, 1}$ and $\|\nabla \theta\|_{\alpha, 1}$ in the advection dispersion equation.

Since

$$
\|\theta\|_{\alpha, 1}>\|\theta\|_{\alpha, 1}-\left\|\theta_{0}\right\|_{\alpha, 1},
$$

then

$$
\frac{\|L(\theta)\|_{\alpha, 1}}{\|\theta\|_{\alpha, 1}} \leq \frac{\|L(\theta)\|_{\alpha, 1}}{\|\theta\|_{\alpha, 1}-\left\|\theta_{0}\right\|_{\alpha, 1}} .
$$

(If $\|\theta\|_{\alpha, 1} \leq\left\|\theta_{0}\right\|_{\alpha, 1}$, then we replace $\|\theta\|_{\alpha, 1}-\left\|\theta_{0}\right\|_{\alpha, 1}$ by $\|\theta\|_{\alpha, 1}-c\left\|\theta_{0}\right\|_{\alpha, 1}$. In fact, $\|\theta\|_{\alpha, 1}>\|\theta\|_{\alpha, 1}-\left\|\theta_{0}\right\|_{\alpha, 1}$ is true under the physical assumption.)

By the definition of the supremum, it is easy to obtain

$$
\|L\|_{\alpha, 1} \leq \frac{\|L(\theta)\|_{\alpha, 1}}{\|\theta\|_{\alpha, 1}-\left\|\theta_{0}\right\|_{\alpha, 1}} .
$$

Since

$$
\|s\|_{\alpha, 1}=\left\|\frac{\partial \theta}{\partial t}+u \cdot \nabla \theta-k \Delta \theta\right\|_{\alpha, 1}=\|L(\theta)\|_{\alpha, 1},
$$

so

$$
\|L\|_{\alpha, 1} \leq \frac{\|s\|_{\alpha, 1}}{\|\theta\|_{\alpha, 1}-\left\|\theta_{0}\right\|_{\alpha, 1}} .
$$

It is clear that

$$
\begin{aligned}
& \|\theta\|_{\alpha, 1} \leq \frac{\|s\|_{\alpha, 1}}{\|L\|_{\alpha, 1}}+\left\|\theta_{0}\right\|_{\alpha, 1} \\
& \leq \frac{\|s\|_{\alpha, 1}}{\sup _{\|\varphi\|_{\alpha, 1}=1}\left\|\frac{\partial \varphi}{\partial t}-k \Delta \varphi+u \cdot \nabla \varphi\right\|_{\alpha, 1}}+\left\|\theta_{0}\right\|_{\alpha, 1} \cdot \\
& \leq \frac{\|s\|_{\alpha, 1}}{|k| \sup _{\|\varphi\|_{\alpha, 1}=1}\|\Delta \varphi\|_{\alpha, 1}-\sup _{\|\varphi\|_{\alpha, 1}=1}\left\|\frac{\partial \varphi}{\partial t}+u \cdot \nabla \varphi\right\|_{\alpha, 1}}+\left\|\theta_{0}\right\|_{\alpha, 1} \\
& \leq \frac{\|s\|_{\alpha, 1}}{|k|_{\|\varphi\|_{\alpha, 1}=1}\|\Delta \varphi\|_{\alpha, 1}-\sup _{\|\varphi\|_{\alpha, 1}=1}\left\|\frac{\partial \varphi}{\partial t}\right\|_{\alpha, 1}-\|u\|_{\alpha, 1} \sup _{\|\varphi\|_{\alpha, 1}=1}\|\nabla \varphi\|_{\alpha, 1}} \\
& \quad+\left\|\theta_{0}\right\|_{\alpha, 1} \cdot
\end{aligned}
$$

By the formula (5), the lower bound for the gradient of 
Compared with the formula (3), the formula (6) gives the error between concentration and average concentration, which has important significance in practice.

$$
\text { Let } I=\{\underbrace{c, c, \cdots, c}_{n}\} \text {, so }\|I \cdot \nabla \theta\|_{\alpha, 1}=c\|\nabla \theta\|_{\alpha, 1} \text {. }
$$

By the formula (1), it is easy to obtain

$$
\frac{\partial \theta}{\partial t}+(u-I) \cdot \nabla \theta+I \cdot \nabla \theta=k \Delta \theta+s .
$$

Since

$$
\begin{aligned}
& \left(c-\|u-I\|_{\alpha, 1}\right)\|\nabla \theta\|_{\alpha, 1} \\
& \leq c\|\nabla \theta\|_{\alpha, 1}-\|(u-I) \cdot \nabla \theta\|_{\alpha, 1} \\
& \leq\|(u-I) \cdot \nabla \theta+I \cdot \nabla \theta\|_{\alpha, 1} \\
& =\|u \cdot \nabla \theta\|_{\alpha, 1}=\left\|k \Delta \theta+s-\frac{\partial \theta}{\partial t}\right\|_{\alpha, 1} \\
& \leq\left\|k \Delta \theta-\frac{\partial \theta}{\partial t}\right\|_{\alpha, 1}+\|s\|_{\alpha, 1},
\end{aligned}
$$

then

$$
\|\nabla \theta\|_{\alpha, 1} \leq \frac{|k| \sup _{\left\|\phi_{1}\right\|_{\alpha, 1} \neq 0} \frac{\left\|\Delta \phi_{1}\right\|_{\alpha, 1}}{\left\|\phi_{1}\right\|_{\alpha, 1}}+\sup _{\left\|\phi_{1}\right\|_{\alpha, 1} \neq 0} \frac{\left\|\frac{\partial \phi_{1}}{\partial t}\right\|_{\alpha, 1}}{\left\|\phi_{1}\right\|_{\alpha, 1}}+\|s\|_{\alpha, 1}}{c-\|u-I\|_{\alpha, 1}} .
$$

The formula (5) and the formula (7) make trouble for us to research the factors on influencing the upper and lower bound for the gradient of concentration in the advection dispersion equation. As we increase the dispersion coefficient, holding the other parameters constant, the lower bound for the gradient of concentration must decrease and the upper bound for the gradient of concentration must increase. One of the reasons for this phenomenon is that the gradient of concentration can be affected by several environmental factors such as temperature, PH, salinity, etc. (see, [16-20]).

By formula (3) and formula (6), we obtain the upper and lower bound for the concentration in the advection dispersion Equation (8).

By the formula (5) and formula (7), we obtain the up- per and lower bound of the gradient of concentration.

$$
\begin{aligned}
& c\|s\|_{\alpha, 1} \\
& \overline{\sup _{\|\phi\|_{\alpha, 1}=1}\left\|\frac{\partial \phi}{\partial t}\right\|_{\alpha, 1}+|u|_{\alpha, 1} \sup _{\|\phi\|_{\alpha, 1}=1}\|\nabla \phi\|_{\alpha, 1}+|k| \sup _{\|\phi\|_{\alpha, 1}=1}\|\Delta \phi\|_{\alpha, 1}} \\
& \leq\|\nabla \theta\|_{\alpha, 1} \leq \frac{|k| \sup _{\left\|\phi_{1}\right\|_{\alpha, 1} \neq 0} \frac{\left\|\Delta \phi_{1}\right\|_{\alpha, 1}}{\left\|\phi_{1}\right\|_{\alpha, 1}}+\sup _{\left\|\phi_{1}\right\|_{\alpha, 1} \neq 0} \frac{\left\|\frac{\partial \phi_{1}}{\partial t}\right\|_{\alpha, 1}}{\left\|\phi_{1}\right\|_{\alpha, 1}}+\|s\|_{\alpha, 1}}{c-\|u-I\|_{\alpha, 1}} .
\end{aligned}
$$

An efficient mixing configuration would have small concentration $\|\theta\|_{\alpha, 1}$ for a given source term and stirring term, indicating a steady state with small variations in the concentration. In general we expect that increasing source term at fixed stirring term should augment concentration.

For the concentration, we focus on the formula (8). As we increase the source amplitude, holding the other parameters constant, the concentration $\|\theta\|_{\alpha, 1}$ must eventually increase. However large concentration does not necessarily imply large source term, as the difference can be made up by the dispersion coefficient $k$ or stirring term. This is what makes enhanced mixing possible. An increase of $\|\theta\|_{\alpha, 1}$ implies that the scalar is more poorly mixed. Formula (8) reflects that we can postpone the increasing of lower bound of concentration by raising the stirring term.

For the gradient of concentration, we focus on the formula (9). As we increase the source amplitude, holding the other parameters constant, the gradient of concentration $\|\nabla \theta\|_{\alpha, 1}$ must eventually increase. However large gradient of concentration does not necessarily imply large source term, as the difference can be made up by other factors. Formula (9) also reflects that we can postpone the increasing of the upper and lower bound of gradient of concentration by raising the stirring term.

By the formula (8) and (9), the concentration $\|\theta\|_{\alpha, 1}$ and the gradient of concentration $\|\nabla \theta\|_{\alpha, 1}$ seem to have the same lower bound, which does not imply that the

$$
\begin{aligned}
& \frac{\|s\|_{\alpha, 1}}{\sup _{\|\phi\|_{\alpha, 1}=1}\left\|\frac{\partial \phi}{\partial t}\right\|_{\alpha, 1}+|u|_{\alpha, 1} \sup _{\|\phi\|_{\alpha, 1}=1}\|\nabla \phi\|_{\alpha, 1}+|k|_{\|\phi\|_{\alpha, 1}=1}\|\Delta \phi\|_{\alpha, 1}} \leq\|\theta\|_{\alpha, 1} \\
& \leq \frac{\|s\|_{\alpha, 1}}{|k| \sup _{\|\varphi\|_{\alpha, 1}=1}\|\Delta \varphi\|_{\alpha, 1}-\sup _{\|\varphi\|_{\alpha, 1}=1}\left\|\frac{\partial \varphi}{\partial t}\right\|_{\alpha, 1}-\|u\|_{\alpha, 1} \sup _{\|\varphi\|_{\alpha, 1}=1}\|\nabla \varphi\|_{\alpha, 1}}+\left\|\theta_{0}\right\|_{\alpha, 1} \text {. }
\end{aligned}
$$


formula (8) and formula (9) are wrong. By the poincare's inequality, the concentration $\|\theta\|_{\alpha, 1}$ and the gradient of concentration $\|\nabla \theta\|_{\alpha, 1}$ must have the same form of the lower bound.

Although our results are established on the unit ball $B_{n}$ in $C^{n}$, our results are obviously correct for any bounded domain $\Omega$ in $C^{n}$. Since $R^{n} \subseteq C^{n}$, so the results in this paper are correct for any bounded domain in $R^{n}$. By the formula (8), if $s \in V M O_{\alpha}^{1}$ and the average function $\hat{s}$ tends zero, then the lower bound of concentration in advection dispersion equation must eventually tend zero and at the same time the upper bound of concentration tends the average concentration of the whole research domain. By the formula (9), if $s \in V M O_{\alpha}^{1}$ and the average function $\hat{s}$ tends zero, then the lower bound for the gradient of concentration in advection dispersion equation must eventually tend zero and at the same time the upper bound for the gradient of concentration does not necessarily tend zero.

By the formulas (8) and (9), we have freedom to choose $\phi$ and calculate its $L^{1}\left(B_{n}, \mathrm{~d} v_{\alpha}\right)$ integral for optimizing the upper and lower bound for the concentration and the gradient of concentration. There is a long way to go before we have satisfactory results.

If $\phi$ and $\varphi$ are time-dependent and still satisfy the formula (8), then we obtain the following result, namely,

$$
\begin{aligned}
& \frac{\|s\|_{\alpha, 1}}{|u|_{\alpha, 1} \sup _{\|\phi\|_{\alpha, 1}=1}\|\nabla \phi\|_{\alpha, 1}+|k|_{\|\phi\|_{\alpha, 1}=1}\|\Delta \phi\|_{\alpha, 1}} \leq\|\theta\|_{\alpha, 1} \\
& \leq \frac{\|s\|_{\alpha, 1}}{\mid k \sup _{\|\varphi\|_{\alpha, 1}=1}\|\Delta \varphi\|_{\alpha, 1}-\|u\|_{\alpha, 1} \sup _{\|\varphi\|_{\alpha, 1}=1}\|\nabla \varphi\|_{\alpha, 1}}+\left\|\theta_{0}\right\|_{\alpha, 1} .
\end{aligned}
$$

If $\phi_{1}$ and $\phi$ are time-dependent and still satisfy the formula (9), then we obtain the following result, namely,

$$
\begin{aligned}
& \frac{c\|s\|_{\alpha, 1}}{|u|_{\alpha, 1} \sup _{\|\phi\|_{\alpha, 1}=1}\|\nabla \phi\|_{\alpha, 1}+|k|_{\|\phi\|_{\alpha, 1}=1}\|\Delta \phi\|_{\alpha, 1}} \leq\|\nabla \theta\|_{\alpha, 1} \\
& \leq \frac{|k|_{\left\|\phi_{1}\right\|_{\alpha, 1} \neq 0} \frac{\left\|\Delta \phi_{1}\right\|_{\alpha, 1}}{\left\|\phi_{1}\right\|_{\alpha, 1}}+\|s\|_{\alpha, 1}}{c-\|u-I\|_{\alpha, 1}} .
\end{aligned}
$$

\section{Conclusions}

It is encouraging that we may obtain the upper and lower bound for the dispersion coefficient $|k|$ and $\|\Delta \theta\|_{\alpha, 1}$ using the same method under the conditions that concentration, source term and stirring term are the control parameters. As a physically meaningful measure of mixing efficiency, we introduce the equivalent diffusivity $k_{e q}$ in [21-23], namely, $k_{e q}^{2}=\frac{k^{2}\left\|\vartheta_{0}\right\|_{\alpha, 1}^{2}}{\|\theta\|_{\alpha, 1}^{2}}$, where $\vartheta_{0}$ is the solution of the advection dispersion equation with the same source but no stirring term. By the upper and lower bound of dispersion coefficient, it is easy to obtain the upper and lower of the equivalent diffusivity. The effective diffusivity is defined in terms of a large-scale gradient of the concentration, whereas here we use the amplitude of the source term, which makes more sense in the present context. In closing we note that all of our analysis, as well as the general result that the equivalent diffusivity depends on the source distribution being smooth enough to have a finite variance. Point sources, for example where $s \sim \delta(z)$, may be of interest in applications but do not have finite variance. In this situation we may still define the mixing efficiency and an equivalent diffusivity via $k_{e q}=k \frac{\left\|\vartheta_{0}\right\|_{\alpha, 1}}{\left\|\vartheta_{0}\right\|_{\alpha, 1}}$. Although we provide a new way to illuminate the quantitative relation among the concentration, dispersion coefficient and gradient of concentration by using BMO theory, it is clear that the concentration, dispersion coefficient and gradient of concentration in advection dispersion equation can be affected by several environment factors(for example [24-29]). So how to fully consider the influence factors on the concentration, dispersion coefficient and gradient of concentration is the key for proceeding the subsequent job.

The investigation of these works is left for future work.

\section{REFERENCES}

[1] H. Aref, "Stirring by Chaotic Advection," Journal of Fluid Mechanics, Vol. 143, No. 1, 1984, pp. 1-21. http://dx.doi.org/10.1017/S0022112084001233

[2] J. M. Ottino, "The Kinematics of Mixing: Stretching, Chaos, and Transprot,” Cambridge University Press, Cambridge, 1989.

[3] J. Schumacher, K. R. Sreenivasan and P. K. Yeung, "Schmidt Number Dependence of Derivative Moments for Quasi-Static Straining Motion,” Journal of Fluid Mechanics, Vol. 479, No. 1, 2003, pp. 221-230. http://dx.doi.org/10.1017/S0022112003003756

[4] J. L. Thiffeault, C. R. Doering and J. D. Gibbon, “A Bound on Mixing Efficiency for the Advection-Diffusion Equation,” Journal of Fluid Mechanics, Vol. 521, No. 1, 2004, pp. $105-114$. http://dx.doi.org/10.1017/S0022112004001739

[5] P. V. Danckwerts, "The Definition and Measurement of Some Characteristics of Mixtures," Applied Scientific Research, Section A, Vol. 3, No. 4, 1952, pp. 279-296.

[6] H. Rehab, R. A. Antonia, L. Djenidi and J. Mi, “Characteristics of Fluorescein Dye and Temperature Fluctuations 
in a Turbulent Near-Wake," Experiments in Fluids, Vol. 28, No. 5, 2000, pp. 462-470. http://dx.doi.org/10.1007/s003480050406

[7] W. Rudin, "Function Theory in the Unit Ball of $C^{n}$," Springer-Verlage, New York, 1980. http://dx.doi.org/10.1007/978-1-4613-8098-6

[8] J. Garnett, "Bounded Analytic Functions," Academic Press, New York, 1981.

[9] F. John and L. Nirenberg, "On Functions of Bounded Mean Oscillation," Communications on Pure and Applied Mathematics, Vol. 14, No. 3, 1961, pp. 415-426. http://dx.doi.org/10.1002/cpa.3160140317

[10] D. Békollé, C. A. Berger, L. A. Coburn and K. H. Zhu, "BMO in the Bergman Metric on Bounded Symmetric Domains,” Journal of Functional Analysis, Vol. 93, No. 2, 1990, pp. 310-350. http://dx.doi.org/10.1016/0022-1236(90)90131-4

[11] C. A. Berger, L. A. Coburn and K. H. Zhu, "BMO on the Bergman Spaces of the Classical Domains," Bulletin of the American Mathematical Society, Vol. 17, No. 1, 1987, pp. 133-136. http://dx.doi.org/10.1090/S0273-0979-1987-15539-X

[12] K. H. Zhu, "BMO and Hankel Operators on Bergman Spaces,” Pacific Journal of Mathematics, Vol. 155, No. 2, 1992, pp. 377-395. http://dx.doi.org/10.2140/pjm.1992.155.377

[13] K. H. Zhu, "Spaces of Holomorphic Functions in the Unit Ball,” Springer-Verlage, New York, 2004.

[14] K. Zhang, C. M. Liu and Y. F. Lu, “Toeplitz Operators with BMO Symbols on the Weighted Bergman Space of the Unit Ball," Acta Mathematica Sinica, English Series, Vol. 27, No. 6, 2011, pp. 2129-2142. http://dx.doi.org/10.1007/s10114-011-0038-3

[15] K. E. Petersen, "Brownian Motion, Hardy Spaces and Bounded Mean Oscillation,” Cambridge University Press, Cambridge, 1977. http://dx.doi.org/10.1017/CBO9780511662386

[16] M. Rosso, J. F. Gouyet and B. Sapoval, "Determination of Percolation Probability from the Use of a Concentration Gradient," Physical Review B, Condensed Matter, Vol. 32, No. 9, 1985, pp. 6053-6054. http://dx.doi.org/10.1103/PhysRevB.32.6053

[17] V. Markin, T. Tsong, R. Astumian and B. Robertson, "Energy Transduction between a Concentration Gradient and an Alternating Electric Field," The Journal of Chemical Physics, Vol. 93, No. 7, 1990, pp. 5062-5066. http://dx.doi.org/10.1063/1.458644

[18] F. Stümpel and K. Jungermann, "Sensing by Intrahepatic Muscarinic Nerves of a Portal-Arterial Glucose Concentration Gradient as a Signal for Insulin-Dependent Glucose Uptake in the Perfused Rat Liver," FEBS Letters,
Vol. 406, No. 1, 1997, pp. 119-122.

[19] A. Lasia, "Porous Electrodes in the Presence of a Concentration Gradient,” Journal of Electroanalytical Chemistry, Vol. 428, No. 1, 1997, pp. 155-164.

[20] M. Higa, A. Tanioka and K. Miyasaka, "Simulation of the Transport of Ions against Their Concentration Gradient across Charged Membranes,” Journal of Membrane Science, Vol. 37, No. 3, 1988, pp. 251-266. http://dx.doi.org/10.1016/S0376-7388(00)82432-1

[21] M. B. Isichenko, "Percolation, Statistical Topography, and Transport in Random Media," Reviews of Modern Physics, Vol. 64, No. 4, 1992, pp. 961-1043.

http://dx.doi.org/10.1103/RevModPhys.64.961

[22] S. B. Pope, “Turbulent Flow," Cambridge University Press, Cambridge, 2000. http://dx.doi.org/10.1017/CBO9780511840531

[23] N. J. Balmforth, W. R. Young, J. Fields, J. L. Thiffeault and C. Pasquero, "Stirring and Mixing: 1999 Program of Summer Study in Geophysical Fluid Dynamics,” Woods Hole Oceanographic Institution, 2000. http://dx.doi.org/10.1575/1912/94

[24] B. Gaylord and S. D. Gaines, "Temperature or Transport Range Limits in Marine Species Mediated Solely by Flow,” The American Naturalist, Vol. 155, No. 6, 2000, pp. 769-789. http://dx.doi.org/10.1086/303357

[25] N. Margvelashvily, V. Maderich and M. Zheleznyak, "THREETOX - A Computer Code to Simulate Three-Dimensional Dispersion of Radionuclides in Stratified Water Bodies,” Radiation Protection Dosimetry, Vol. 73, No. 1-4, 1997, pp. 177-180.

http://dx.doi.org/10.1093/oxfordjournals.rpd.a032128

[26] D. T. Ho, P. Schlosser and T. Caplow, "Determination of Longitudinal Dispersion Coefficient and Net Advection in the Tidal Hudson River with a Large-Scale, High Resolution SF6 Tracer Release Experiment," Environmental Science and Technology, Vol. 36, No. 15, 2002, pp. 32343241. http://dx.doi.org/10.1021/es015814+

[27] G. H. O. Essink, "Salt Water Intrusion in a Three-Dimensional Groundwater System in the Netherlands: A Numerical Study,” Transport in Porous Media, Vol. 43, No. 1, 2001, pp. 137-158. http://dx.doi.org/10.1023/A:1010625913251

[28] E. Sierra, F. G. Acien, J. M. Fernandez, J. L. Garcia, C. Gonzalez and E. Molina, "Characterization of a Flat Plate Photobioreactor for the Production of Microalgae,” Chemical Engineering Journal, Vol. 138, No. 1, 2008, pp. 136-147. http://dx.doi.org/10.1016/j.cej.2007.06.004

[29] L. Y. Chang and W. C. Chen, "Data Mining of TreeBased Models to Analyze Freeway Accident Frequency,” Journal of Safety Research, Vol. 36, No. 4, 2005, pp. 365375. http://dx.doi.org/10.1016/j.jsr.2005.06.013 